\title{
Dictynna
}

Dictynna

Revue de poétique latine

$8 \mid 2011$

Varia

\section{Zoophilie in Zoologie und Roman: Sex und Liebe zwischen Mensch und Tier bei Plutarch, Plinius dem Älteren, Aelian und Apuleius}

\section{Judith Hindermann}

\section{(2) OpenEdition \\ Journals}

Édition électronique

URL : https://journals.openedition.org/dictynna/717

DOI : $10.4000 /$ dictynna.717

ISSN : $1765-3142$

Éditeur

Université de Lille

\section{Référence électronique}

Judith Hindermann, « Zoophilie in Zoologie und Roman: Sex und Liebe zwischen Mensch und Tier bei Plutarch, Plinius dem Älteren, Aelian und Apuleius », Dictynna [En ligne], 8 | 2011, mis en ligne le 23 septembre 2011, consulté le 21 septembre 2021. URL : http://journals.openedition.org/dictynna/717 ; DOl : https://doi.org/10.4000/dictynna. 717

Ce document a été généré automatiquement le 21 septembre 2021.

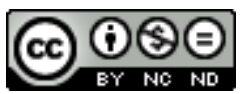

Les contenus des la revue Dictynna sont mis à disposition selon les termes de la Licence Creative Commons Attribution - Pas d'Utilisation Commerciale - Pas de Modification 4.0 International. 


\title{
Zoophilie in Zoologie und Roman: Sex und Liebe zwischen Mensch und Tier bei Plutarch, Plinius dem Älteren, Aelian und Apuleius ${ }^{1}$
}

\author{
Judith Hindermann
}

1 Sex mit einem Tier - ein Tatbestand, der bis Ende des 18. Jahrhunderts in fast allen europäischen Staaten mit dem Tode bestraft und weit ins 20. Jahrhundert durch mehrjährige Gefängnisstrafen geahndet wurde - gilt, obwohl in den meisten Ländern Europas inzwischen straffrei, ${ }^{2}$ als das letzte Tabu in Sachen Sexualität und bleibt sowohl in der öffentlichen Diskussion als auch in der erzählenden zeitgenössischen Literatur weitgehend ausgespart. ${ }^{3}$ Auch in der klassischen Philologie wurde das Thema ausserhalb des Kontexts von Mythos und Kult nur selten aufgegriffen. ${ }^{4}$ Es fehlt in vielen Studien zur Sexualität, ${ }^{5}$ zur bildlichen Darstellung von Sexualität ${ }^{6}$ oder zum Tier bzw. zur Zoologie in der Antike. ${ }^{7}$

2 Überblickt man die überlieferten literarischen Texte, fällt auf, dass Zoophilie ausserhalb des Mythos vor allem in zwei literarischen Gattungen auftritt : Einerseits in den zoologischen Schriften von Plutarch, Plinius dem Älteren und Aelian, andererseits im antiken Roman, d.h. in den Metamorphosen des Apuleius und im pseudo-lukianischen Onos. In diesem Beitrag soll untersucht werden, welche Funktion Zoophilie in den beiden literarischen Gattungen hat und wie die Autoren die sexuelle Praktik bewerten, die weder nach griechischem noch römischen Recht strafbar war. ${ }^{8}$ Da Vorstellungen über Tiere eng mit Gender und Geschlechterhierarchien verbunden sind, ${ }^{9}$ soll insbesondere die Frage berücksichtigt werden, inwiefern männliche und weibliche Verhaltens- und Rollenzuschreibungen bei der Darstellung zoophiler Akte wirksam werden. Auf die Ikonographie der Zoophilie ${ }^{10}$ oder auf Zoophilie als Straf- und Foltermethode ${ }^{11}$ wird in diesem Beitrag hingegen nicht eingegangen.

3 Für sexuelle Akte bzw. Liebe zwischen Mensch und Tier kennt die Antike keinen eigenen Begriff, sondern verwendet mit amare bzw. દ́pác dieselben Termini wie für 
zwischenmenschliche Kontakte. In diesem Beitrag wird der moderne Begriff „Zoophilie“ benutzt, der gemäss Definition eine emotionale Bindung zu einem Tier bezeichnet, die zu dessen Bevorzugung als Lebensgefährte und/oder Sexualpartner führt. Anders als der Begriff „Sodomie“, der sich nur auf den sexuellen, nicht aber den emotionalen Aspekt bezieht, deckt der Terminus ein breites Spektrum von Kontaktformen zwischen Mensch und Tier ab und schliesst sowohl den Menschen als auch das (dressierte) Tier als Initiator der Beziehung ein, was sich als ein relevanter Faktor bei der Beurteilung von Zoophilie in der antiken Zoologie erweist. ${ }^{12}$

\section{Zoophilie in der antiken Zoologie}

Eine besonders reiche Quelle für Zoophilie sind die zoologischen Schriften von Plutarch, Plinius dem Älteren und Aelian. Anders als im Mythos, wo zumeist eine Frau/ Göttin in menschlicher Gestalt durch einen Gott in Tiergestalt vergewaltigt wird ${ }^{13}$ und in Folge dessen über die Spezies hinweg menschlicher, göttlicher oder tierischer Nachwuchs entsteht ${ }^{14}$ geht es in den bei den antiken Zoologen überlieferten Geschichten nicht nur um Akt und Zeugung, sondern auch um Freundschaft, Liebe, Zuneigung und Verführung zwischen Mensch und Tier. ${ }^{15}$ Ebenfalls anders als im Mythos, wo es sich ausser bei Ganymed stets um heterosexuelle Zoophilie handelt ${ }^{16}$ und die Täter mit Ausnahme der Pasiphaë ${ }^{17}$ immer männlich sind, variieren bei den antiken Zoologen die Perspektiven, aus der die Mensch-Tier-Geschichten erzählt werden, ebenso wie Geschlecht und aktives bzw. passives Rollenverhalten der Beteiligten stärker.

5 Da dieselben Anekdoten über Zoophilie bei Plutarch, Plinius dem Älteren und Aelian in ganz unterschiedlicher Ausprägung erscheinen, dienen sie nicht nur der Charakterisierung der unmittelbar beteiligten Tiere und Personen, sondern lassen sich auch als Ausdruck einer generellen Sicht der Autoren auf die Beziehung zwischen Mensch und Tier interpretieren. Die Grenze zwischen Geschichten, welche die geistige Liebe bzw. Freundschaft zwischen Mensch und Tier respektive einer Tierart belegen sollen, und solchen, die einen dezidiert erotisch-sexuellen Hintergrund haben, ist dabei nicht immer klar zu ziehen. ${ }^{18}$ Diese Unschärfe ist dem antiken Diskurs über das Verhältnis von Mensch und Tier inhärent, der geprägt ist durch ein Nebeneinander von Nähe und Distanz, das als Vorstellung einer Artverwandtschaft gedeutet werden kann und seinen Ausdruck in zahlreichen Mythen über Mischwesen und Monster findet. ${ }^{19}$

Seit dem 5. Jh. v. Chr. lässt sich neben einem oppositionellen Modell des Verhältnisses von Mensch und Tier ein stärker graduell gedachtes Modell feststellen. ${ }^{20}$ Die neue Art, Differenzen - nicht nur zwischen Mensch und Tier, sondern auch zwischen Mann und Frau - zu betrachten, findet sich erstmals bei Aristoteles, dem bedeutendsten Vertreter der antiken Zoologie. Einher mit dieser Vorstellung einer Kontinuität geht in Aristoteles' zoologischen Schriften jedoch gleichzeitig eine klare Grenzziehung zwischen Pflanzen und Tieren sowie Tieren und Menschen. ${ }^{21}$ Anders als in den Schriften von Plutarch, Plinius dem Älteren und Aelian spielt Zoophilie keine Rolle als Form der Interaktion zwischen Mensch und Tier $^{22}$ und die Existenz von Mischwesen hält Aristoteles deshalb für unmöglich, weil sich Lebewesen über die Artgrenze nur dann fortpflanzen können, wenn sie über ähnliche Grösse und Tragzeiten verfügen. ${ }^{23}$ Berichte über Mischwesen, die auf Sex zwischen Mensch und Tier bzw. Tiere 
unterschiedlicher Gattungen schliessen lassen, erklärt er rationalisierend mit der Ähnlichkeit missgestalteter Menschen oder Tiere mit anderen Tieren. ${ }^{24}$

7 Mit „Zoologie“ wird im Folgenden daher nicht die systematisch-wissenschaftliche Behandlung von Morphologie, Anatomie und Physiologie im aristotelischen Sinn bezeichnet, sondern eine Naturgeschichte der Tiere im weitesten Sinn. Charakteristisch für diese Art der Zoologie, die bei späteren griechischen und römischen Schriftstellern vorherrscht, ist das Interesse am Aussergewöhnlichen (paradoxa) und Wundersamen (mirabilia) der Tierwelt ${ }^{25}$ sowie die Wahrnehmung der Tiere in anthropologischen Kategorien. Mensch und Tier begegnen sich in der Beschreibung von Plutarch, Plinius dem Älteren und Aelian auf Augenhöhe und ihr Verhalten wird bei der Beschreibung von Zoophilie entlang denselben, nämlich menschlichen, Vorstellungskategorien gezeichnet und beurteilt. Da die Annäherung bestimmter Tiere an und die Werbung um den geliebten Menschen wie beim Menschen auf einer bewussten, individuellen Entscheidung, und nicht auf dem Instinkt basiert, ${ }^{26}$ ist für die Bewertung von Zoophilie bei allen drei Autoren die Frage zentral, wer die Initiative zur zoophilen Beziehung unternimmt.

\section{Der Mensch als Aggressor : Verurteilung der Zoophilie}

Plutarch gilt aufgrund seiner Schriften De esu carnium, De amore prolis, De sollertia animalium und Bruta animalia ratione uti als erster antiker Denker mit einem sympathetischen Verständnis für Tiere. Seine Werke basieren auf der anti-stoischen Grundhaltung, dass zwischen Mensch und Tier keine prinzipiellen, sondern nur quantitativ seelisch-geistige Differenzen bestehen. ${ }^{27}$ Daraus resultiert für die Menschen die moralische Pflicht zur Gerechtigkeit gegenüber den Tieren, die man sich zwar nutzbar machen darf, jedoch nicht unnötig jagen, quälen oder töten soll (mor. 964E965B). Wie im Dialog Bruta animalia ratione uti deutlich wird, zählt dazu auch die Benutzung eines Tiers zur sexuellen Befriedigung. Zoophilie dient hier dem von Circe in ein Schwein verwandelten Gryllus, der seine menschliche Gestalt zu Odysseus' Erstaunen nicht zurück will, als Beweis für die Verdorbenheit und Perversion der Menschen. ${ }^{28}$ Die unmässige Lust bringe die Männer dazu, die Grenzen der Natur zu überschreiten und sich mit Ziegen, Schweinen und Stuten zu paaren. Auch Frauen haben sich mit Tieren eingelassen und so seien die verschiedenen Mischwesen (Minotauren, Aegipane, Centauren, Sphingen) entstanden (mor. 991A). Die einzelnen Geschichten werden von Gryllus nicht detailliert ausgeführt, sondern nur mit dem Geschlechtsakt bzw. den daraus resultierenden Nachkommen betitelt. Als Auslöser der Taten sieht Gryllus die menschliche Lust, die als widernatürlich bzw. widerrechtlich ( $\pi \alpha \rho \alpha v о \mu о \tilde{\sigma} \sigma v)$ bewertet und der tierischen unverdorbenen Natur gegenübergestellt

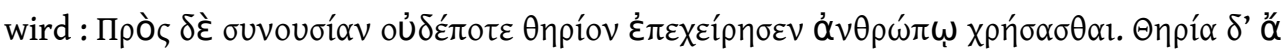

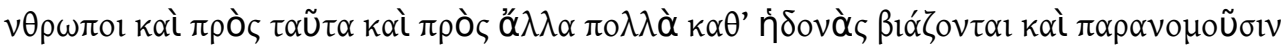
(mor.991A). ${ }^{29}$ Die Tiere dagegen erscheinen in Gryllus' Rede als wehrlose Opfer, die von Natur aus keusch sind und nie einen Menschen aus Gründen der Lust angreifen würden. Ihre besondere Wirkkraft bekommt die Argumentation aus dem Munde eines Schweins, das selbst zu den lüsternen Tierarten zählt und Plutarch dennoch dem Menschen als moralisch überlegen zeigt. ${ }^{30}$

9 Auch in Aelians De Natura Animalium wird die sexuelle Annäherung von Menschen an Tiere in einen negativen Kontext gestellt, indem die Täter für ihre Tat mit dem Leben 
büssen müssen. Die erste der beiden Geschichten, die Aelian erzählt, handelt von einem Stallburschen, der sich in die schönste Stute aus seinem Stall verliebt und mit ihr schläft. Das Fohlen, das die Szene beobachtet, empfindet die Tat als Tyrannei

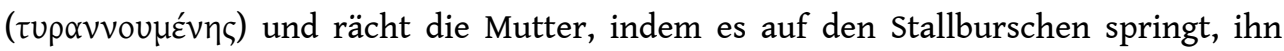
erschlägt und zudem später dessen Grab schändet (NA 4,8). In der zweiten Geschichte geht es um einen Hirtenjungen, Crathis, der die schönste seiner Ziegen liebt, mit Geschenken und Gefälligkeiten um sie wirbt und schliesslich eine sexuelle Beziehung mit ihr eingeht. Der Ziegenbock der Herde erschlägt den Hirten schliesslich aus Eifersucht, indem er dem Schlafenden die Hörner gegen den Schädel rammt (NA 6,42). Beide von Aelian referierten Geschichten enthalten realistische Details : Die Täter sind junge Männer, die aufgrund ihres Berufes engen Umgang mit Tieren und Gelegenheit haben, sich diesen unbeobachtet zu nähern. Dass Hirten mangels Frauen mit ihren Tieren schlafen und sogar Nachkommen zeugen, wird auch bei anderen Autoren als allgemein bekannte Tatsache vorausgesetzt. ${ }^{31}$ Aelian schildert diese Form von Zoophilie dennoch als ein von der Gesellschaft negativ bewertetes Verhalten, indem er neben den Reaktionen der „entehrten Angehörigen“ der geschändeten weiblichen Tiere auch den inneren Konflikt des Stallburschen vor der Tat zeigt, der zunächst versucht, sein

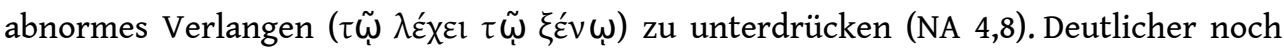
wird die Verurteilung der Zoophilie bei Aelian in der Geschichte des Atheners Socles,

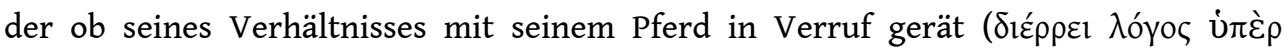
$\dot{\alpha} \mu \varphi \circ \tilde{v} v \dot{\alpha} \tau o \pi \omega ́ \tau \varepsilon \rho \circ \varsigma)$ und das Tier deshalb verkauft (NA 6,44).

Auch Plinius der Ältere erwähnt sexuelle Beziehungen und daraus resultierende Mischwesen zwischen Mensch und Tier in seinem siebten Buch über den Menschen, bezeichnenderweise aber als Phänomen, das nur bei indischen Völkern am Rande der Oikumene vorkommt: Duris Indorum quosdam cum feris coire mixtosque et semiferos esse partus (nat. 7,30). Die babylonische Königin Semiramis, die Sex mit ihrem Pferd gehabt haben soll, ist ebenfalls im entfernten Asien beheimatet. ${ }^{32}$ Monstren und Mischwesen finden sich dagegen nicht nur in Indien, sondern auch auf italischem Boden. ${ }^{33}$ Deren Entstehung stellt Plinius jedoch entgegen der Tradition nicht in den Kontext von Zoophilie. ${ }^{34}$ Als sexuelle Praxis bei den zivilisierten Römern ist Zoophilie in Plinius' Naturalis historia somit nicht existent.

Der Grund, warum vom Menschen initiierte Zoophilie von den Zoologen abgelehnt wird, ist nicht eindeutig zu fassen. Während die Verurteilung der Zoophilie heute wesentlich durch Fragen des Tierschutzes bestimmt ist, ${ }^{35}$ spielt diese Überlegung keine Rolle. Die Vorstellung von Grausamkeit gegenüber den Tieren, die zu Tausenden in der Arena getötet wurden, ${ }^{36}$ ist in der antiken Literatur kaum ausgeprägt und Schutzbestimmungen für Tiere sind selten belegt. ${ }^{37}$ Das Verhalten gegenüber Tieren wird bei Plutarch, Plinius dem Älteren und Aelian auch nicht, anders z.B. als im Diskurs über den Umgang mit Sklaven, ${ }^{38}$ als Hinweis auf den Charakter des Besitzers verstanden. Ein Grund für die Kritik der Zoophilie könnte die Furcht vor dem Entstehen von Mischwesen sein, die als Verstoss gegen die Natur und als schlechte Omina gedeutet werden. Diese Furcht vor den Folgen zoophiler Kontakte wird explizit in Philons Abhandlung über die Tiere aus dem 1. Jh. n. Chr. formuliert ${ }^{39}$ und hält sich bis weit ins 19. Jahrhundert. ${ }^{40}$ 


\section{Das Tier als Verführer : Beweis der tierischen Intelligenz und miraculum} der Fälle Frauen, die zoophile Akte begehen. Entsprechend der traditionellen Geschlechterrollen bleiben die Frauen dabei jedoch passiv und werden vom Tier, das entweder explizit als männlich beschrieben wird oder dessen Geschlecht unbestimmt bleibt, umworben und verführt bzw. vergewaltigt. Analog dazu werden auch Knaben von Tieren verführt, hingegen nur sehr selten erwachsene Männer. Nur in einer Geschichte Aelians (NA 4,54) ist es dezidiert ein weibliches Tier, eine Schlange, die aktiv um einen Knaben wirbt. In der Perspektive des Tieres, das um den geliebten Menschen wirbt, spiegeln sich somit die Werthaltungen und Rollenvorstellungen des männlichen Autors und der Leser, die sich mit dem aktiven Tier identifizieren. Dass es bei den antiken Zoologen in der Mehrzahl der Fälle Tiere, und nicht Menschen sind, welche die Initiative zu zoophilen Akten ergreifen, erklärt sich mit dem speziellen Fokus der Schriftsteller auf Aussergewöhnliches und Wundersames.

Plutarch lässt in seinem Dialog De sollertia animalium, der sich um die Frage dreht, ob Land- oder Wassertiere intelligenter sind, Aristotimus, den Anwalt der Landtiere, mittels vier Geschichten über Zoophilie beweisen, dass das Verhalten bestimmter Tiere dem verliebter Menschen gleicht (mor. 972D-F). In der ersten Geschichte wirbt ein Elefant um eine Kranzverkäuferin, indem er ihr Früchte mitbringt, sich neben sie stellt und sie mit seinem Rüssel am Busen berührt. ${ }^{41}$ In der zweiten Geschichte wird eine Frau aus Aeolien jede Nacht von einer Schlange besucht, die sich unter sie schiebt und Haut an Haut zu liegen kommt. ${ }^{42}$ Auf eine von den Verwandten der Frau erzwungene Trennung reagiert die Schlange, indem sie sich nach der Wiedervereinigung eng um die Frau schlingt, ihr dadurch die Hände am Körper festbindet und dann die Gefesselte zur Bestrafung mit dem Schwanzende auf die Waden schlägt.

Der Fokus des Erzählers liegt in den beiden ausführlicher beschriebenen Fällen ${ }^{43}$ ganz auf dem Verhalten der anthropomorph beschriebenen Tiere. Elefant und Schlange werben aktiv um die geliebten Frauen und suchen ihre Gefühle gleich den Menschen, aber mit den ihnen eigenen Mitteln auszudrücken, indem sie statt den Händen Rüssel und Schwanzende, statt Worten die Körpersprache nutzen. Wie die Frauen auf die Avancen des Tieres reagieren, ob sie dessen Gefühle teilen, als unangenehm empfinden oder überhaupt als Liebe wahrnehmen, interessiert den Erzähler nicht. Die Frauen erscheinen als stumme Objekte, an denen die Tiere ihren Verstand demonstrieren können. Zoophilie dient Plutarch, wie andere komplexere Handlungen auch, ${ }^{44}$ als Beweis dafür, dass Tiere über Intelligenz verfügen, die darin besteht, menschliches, d.h. männlich-aktives Werbeverhalten zu adaptieren. Das Fehlen der Hände, mit dem Anaxagoras den Mangel an Kultur bei den Tieren erklärt, ${ }^{45}$ wird hier nicht als Beweis für die fehlende Intelligenz der Tiere angeführt, sondern erst recht als Indiz für dieselbe gewertet, da die Tiere zwecks erotischer Werbung ihre naturgegebene Beeinträchtigung mit anderen Körperteilen zu kompensieren vermögen.

Wie Plutarch führt auch Plinius das Thema Zoophilie mit der Anekdote über die Liebe eines Elefanten zu einer Kranzverkäuferin ${ }^{46}$ ein und fügt dann zwei weitere Fälle an, in denen sich ein Elefant in eine junge Frau (eine Salbenverkäuferin) oder einen jungen Mann (einen griechischen Soldaten) verliebt. Auffällig ist die Zurückhaltung, mit der Plinius die Anekdoten referiert. Das bei Plutarch geschilderte Detail etwa, dass der 
Elefant die Brust seiner Angebeteten mit dem Rüssel streichelt, fehlt. Plinius' Elefanten äussern ihre Zuneigung durch Freude beim Anblick des oder der Geliebten, durch Hungerstreik bei deren Abwesenheit, ungeschickte Liebkosungen sowie Geschenke (nat. 8,13-14). Auch andere bekannte Geschichten über die Liebe von Gänsen (nat. 10,51) und Delphinen (nat. 9,25-27) zu Menschen schreibt Plinius so um, dass nicht die erotische Anziehung, sondern die Freundschaft und der (moralische) Nutzen der Tiere für den Menschen ins Zentrum gerückt werden. ${ }^{47}$ Gänse etwa helfen dem Menschen durch ihre Wachsamkeit und Weisheit, ebenso ist der Delphin, der für Fische gefährlichste Feind, ein Menschenfreund (homini ... amicum nat. 9,24), der den Fischern mutig und diszipliniert beim Fischfang hilft (nat. 9,29-32).

Die als natürlich gelobte tierische Sexualität setzt Plinius in seiner Naturalis historia wiederholt der durch den Verstand pervertierten menschlichen Sexualität entgegen. ${ }^{48}$ Erstere zeichnet sich durch Mässigung und Ausrichtung auf die Fortpflanzung aus. ${ }^{49}$ Um die These von der moralisch vorbildlichen Wirkung von Tieren auf die Menschen nicht zu entkräften, müssen die tradierten Geschichten über Zoophilie, die vom Tier aus geht, entsprechend Plinius' didaktischer Intention entschärft werden. Plinius tut dies, indem er zum einen erotische oder sexuell explizite Details weglässt und zum andern die Geschichten mit den positiven charakterlichen Eigenschaften der Tiere einrahmt.

In Aelians De Natura Animalium spielt das Aussergewöhnliche, Paradoxe (paradoxa) und Wundersame (mirabilia), das primär der Unterhaltung dient, gegenüber dem Anspruch auf Belehrung der Leserschaft eine weit grössere Rolle als bei Plutarch und Plinius. ${ }^{50}$ Entsprechend nimmt auch die Zoophilie bei Aelian grossen Raum ein : Er berichtet über Zoophilie mit insgesamt elf verschiedenen Tierarten, ${ }^{51}$ indem er in Übersichtskapiteln die Geschichten verschiedener Tieren aufzählt, die sich in Frauen oder Knaben verliebt haben. ${ }^{52}$ Zoophilie erscheint auch in Zusammenhang mit der Beschreibung besonderer charakterlicher Eigenschaften von Tieren. Als lüstern und zügellos ( $\alpha$ kó $\lambda \alpha \sigma \tau \alpha)$ gelten Hunde, Paviane und Geissböcke, von denen man sagt, dass sie mit Frauen schlafen oder diese vergewaltigen..$^{53}$

Neben katalogartigen Sammelkapiteln zum Thema Zoophilie bietet Aelian auch verschiedene ausführlicher erzählte Belege, so die Geschichte über eine Schlange und einen Rinderhirten (NA 8,11), eine Schlange und eine Frau aus Judaea (NA 6,17) sowie den Athener Socles und sein Pferd (NA 6,44). Aelians Fokus ist dabei ein zweifacher: Erstens werden die Anekdoten mit zusätzlichen erotisch-sexuellen Details versehen. Die Schlangen etwa, die sich in den Hirten und in die Frau verlieben, sind enorm gross

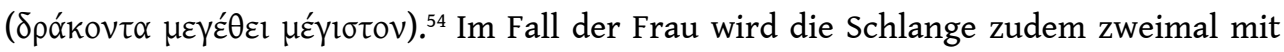

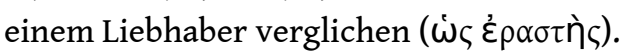

Zweitens dienen die Geschichten Aelian als Beweis für die Macht Amors, dem auch die Tiere hilflos ausgeliefert sind. Dies führt zum Widerspruch, dass Aelian den Tieren einerseits wie Plutarch und Plinius moralische Qualitäten und Leistungen zuschreibt, die über diejenigen der Menschen hinausgehen - indem er z.B. ein Inzest-Tabu schildert, das Tiere angeblich strenger als Menschen befolgen, oder auf die tierische Nächstenliebe oder den Selbstmord aus Scham verweist,$-{ }^{55}$ gleichzeitig aber unkritisch über Tiere berichtet, die mit Menschen erotische oder sexuelle Beziehungen eingehen wollen. Aelian führt als Erklärung, und damit implizit auch als Entschuldigung die allumfassende Macht des Liebesgottes an, der nicht nur über alle anderen Götter, sondern auch über die Tiere herrscht, die als unvernünftig ( $\left.\tau \tilde{\omega} v \alpha \dot{\alpha} \lambda{ }^{\prime} \gamma \omega v\right)$ beschrieben 
werden. ${ }^{56}$ Wie in den griechischen Liebesromanen, ${ }^{57} \mathrm{zu}$ denen Aelian eine grosse Nähe aufweist, spielt neben Amor auch die Schönheit der Beteiligten eine zentrale Rolle, weil sie via Auge Liebe auf den ersten Blick auslöst. Tiere, insbesondere die scharfsichtige Schlange (NA 8,11) und der intelligente Delphin (NA 6,15), sind in Aelians Darstellung fähig, menschliche Schönheit zu erkennen und zu würdigen..$^{58}$ Gleichzeitig macht die Schönheit die Ebenbürtigkeit der Verliebten öffentlich sichtbar, wie in der Geschichte über Socles und sein Pferd deutlich wird, die beide von ihrer Umgebung als attraktiv und klug wahrgenommen werden (NA 6,44). Durch die Betonung der Schönheit der Beteiligten und des Einflusses des Liebesgottes auf das Entstehen einer zoophilen Beziehung wird bei Aelian das miraculum unterstrichen und gleichzeitig die Grenze zwischen Mensch und Tier verwischt.

19 Überblickt man die Geschichten in ihrer Gesamtheit, zeigt sich, dass Zoophilie bei den drei Zoologen je nachdem, wer die Initiative zur zoophilen Beziehung ergreift, unterschiedliche Bewertung erfährt. Aus Sicht des passiv bleibenden Tieres sind die menschlichen Annäherungen bei Plutarch, Plinius dem Älteren und Aelian gleichermassen Zeichen von perverser Lust bzw. Barbarei und werden als sexuelle Übergriffe beschrieben, die durch Mischwesen und Monstren in der Öffentlichkeit manifest werden. Zoophilie dient allen drei Autoren als Beweis für die Verdorbenheit der Menschen, deren degeneriertem Verhalten die als natürlich gepriesene Sexualität der Tiere vorbildlich gegenübergestellt wird. Dagegen wird Zoophilie, die vom Tier ausgeht, von den Zoologen je nach Intention ihres Werks zu unterschiedlichem argumentativem oder narrativem Zweck angeführt, erfährt jedoch in keinem Fall explizite oder implizite Kritik. Die Vorstellung von der natürlichen Unschuld und artgemässen Handlungsweise von Tieren ${ }^{59}$ erweist sich als die dominierende Sichtweise auf jegliches tierische Verhalten.

\section{Liebestolle Frauen : Zoophilie im antiken Roman}

Dass sich Frauen selbst aktiv einem Tier in zoophiler Absicht nähern, ist in den zoologischen Schriften ebenso wie im Mythos der Sonderfall, im Kontext von Satire und Invektive dagegen die Regel. Juvenal (6,332-334) schildert Frauen, die sich in rasender Lust mit Eseln vereinigen, wenn gerade keine freien Männer oder Sklaven zur Verfügung stehen, ${ }^{60}$ und Prokop (hist. 9,20-21) beschreibt in seinen Anekdota, dass sich die Kaiserin Theodora auf offener Bühne von Gänsen Körner aus dem Schoss picken liess. Beiden Autoren dienen diese Beispiele als Kulminationspunkt ihrer Beweisführung der Verdorbenheit und sexuellen Besessenheit von Frauen. Zoophilie ist - ähnlich wie das Ausüben anderer Praktiken wie Cunnilingus oder Fellatio ${ }^{61}$ - ein Vorwurf, der zur Invektive gegen Individuen oder das ganze weibliche Geschlecht verwendet wird.

21 Auch im antiken Roman sind es ausschliesslich Frauen, die sich in zoophiler Absicht Tieren, ebenfalls Eseln und Gänsen, nähern, ${ }^{62}$ erstaunlicherweise werden sie in dieser literarischen Gattung jedoch weder explizit für ihr Verhalten kritisiert noch wie bei Aelian durch Tod oder Unglück implizit bestraft. In Apuleius' Metamorphosen (met. 10,19,3-22,5), die ausführlichste Darstellung von Zoophilie überhaupt in der antiken Literatur, fällt dies deshalb besonders auf, weil sich Apuleius' Protagonist Lucius ansonsten betont gegen Ehebruch und unmoralisches Verhalten ausspricht und öffentliche Zoophilie in der Arena als äusserst entehrende Strafe beschreibt (met. 
10,29.34). Anders als im Onos, der Epitome der verlorenen griechischen Metamorphosen, auf denen Apuleius' Metamorphosen basieren, wäre bei Apuleius eine Bestrafung der zoophilen matrona - analog zu der anderer Personen im Roman, die durch voluptas motiviert gegen Sitte und Gesetz verstossen - ohne Weiteres möglich. ${ }^{63}$ Apuleius legt den Fokus der Geschichte jedoch nicht auf moralische Fragen, sondern auf die fliessende Grenze zwischen Mensch und Tier und die Umkehr der traditionellen Geschlechterrollen, indem er den Esel Lucius als käuflichen Liebessklaven auftreten lässt. ${ }^{64}$

Ganz zu Beginn der Geschichte bezeichnet Apuleius die matrona als asinaria Pasiphaae, als „Pasiphaë mit einem Esel““. ${ }^{65}$ Dadurch evoziert er den Mythos der „Ahnherrin“ der Zoophilie, vor dessen Folie die aktuelle Geschichte gelesen werden soll, und gibt dem gebildeten Leser, ähnlich wie er es auch an anderer Stelle tut, ${ }^{66}$ ein Signal, das ihn auf intertextuelle Bezüge aufmerksam machen soll. Ursprünglich Mythos einer Bestrafung und Erniedrigung des Königs Minos durch den Gott Poseidon, verlagert sich in der literarischen Tradition der Schwerpunkt der Erzählung auf Pasiphaë, die sich vom passiven Opfer in eine aktive Täterin verwandelt. Damit einhergehend wird der komische Aspekt der Erzählung, Pasiphaës Werbung um den desinteressierten Stier, hervorgehoben, und sowohl der Götterzorn, der das Verhalten der Königin erklärt, als auch das technische Konstrukt des Dädalus, das die praktische Umsetzung plausibel macht, in den Hintergrund gerückt.

Erste Ansätze für eine solche Umwertung des Mythos finden sich bei Vergil, der ihn in seinen Eclogen $(6,45-60)$ ins bukolische Milieu ${ }^{67}$ transportiert und die verheiratete Königin in ein unglückliches Hirtenmädchen (virgo infelix 47) verwandelt. ${ }^{68}$ Ovid entwickelt Vergils Ansatz, Pasiphaë als verliebte Frau, und nicht als mythisches Opfer göttlichen Zorns darzustellen, in seiner Ars amatoria (1,289-326) weiter, ${ }^{69}$ indem er die komische Inkongruenz im Handeln der Pasiphaë hervorhebt, ${ }^{70}$ die versucht, dem Stier durch kostbare Kleidung und Frisur zu gefallen (303-307), und sich ihrer tierischen Nebenbuhlerinnen raffiniert entledigt (313-322). In der psychologischen Deutung des praeceptor amoris erscheint Pasiphaë als extremer Fall der furiosa libido (281), die allen Frauen eigen ist, und dient als Argument dafür, dass der Liebesschüler darauf vertrauen kann, in seinem Werben nicht zurückgewiesen zu werden. ${ }^{71}$

Auch Apuleius' Lucius trifft auf eine Pasiphaë, die wie die exempla aus Ovids Ars amatoria von einer rasenden Lust (vaesana libido met. 10,19,3), aber nicht auf einen Stier, sondern auf einen Esel ergriffen ist. Gleichzeitig wird jedoch der Blickwinkel, aus dem die Geschichte erzählt wird, durch Lucius' Ankündigung verändert. Während in der traditionellen Überlieferung des Pasiphaë-Mythos das Verlangen der Frau aus einer auktorialen Perspektive geschildert wird und das Tier stumm bleibt, werden bei Apuleius die Geschehnisse aus der Perspektive des menschlichen Ich-Erzählers und männlichen Esels geboten. ${ }^{72}$ Die Grenze zwischen Mensch und Tier wird nicht nur durch diese Personalunion von Esel und Erzähler, sondern auch durch das Verhalten der matrona verwischt. Diese wählt wie Ovids Pasiphaë Mittel, die dazu geeignet sind, einen Mann, nicht ein Tier zu verführen, indem sie das Schlafzimmer mit Kissen, Decken und Kerzen schön einrichtet, sich und den Esel mit wohlriechendem öl parfümiert und ihn mit Küssen bedeckt (met. 10,20,1-2). Insbesondere die Sprache spielt bei der Verführung des Esels in Apuleius' Darstellung eine grosse Rolle, obwohl die matrona damit rechnen muss, dass er sie gar nicht versteht. 

wesentliches Distinktionsmerkmal des Menschen gegenüber dem Tier. ${ }^{73}$ Speziell Liebesworte gehören zum angeborenen Grundwissen der menschlichen Spezies ${ }^{74}$ und sind für das Entstehen der Liebe von grosser Bedeutung. ${ }^{75}$ Während im Onos nur vermerkt wird, dass sich die matrona mit dem Esel wie mit einem menschlichen Liebhaber unterhält, ${ }^{76}$ rapportiert Lucius dem Leser die Liebesbekundungen der Dame im „originalen“ Wortlaut, indem er vier spezifische Ausdrücke nennt und die übrigen als typische Worte verliebter Frauen zusammenfasst : „amo “ et ",cupio“ et „te solum diligo" et "sine te iam vivere nequeo" et cetera, quis mulieres et alios inducunt et suas testantur adfectiones (met. 10,21,3). ${ }^{77}$ Auch während des Akts bezeichnet die matrona den Esel Lucius als palumbulum („Täubchen“) und passer (,Spatz“). Damit befolgt sie einerseits Ovids Rat an seine Liebesschülerinnen, dem Partner während des Akts Koseworte zuzuflüstern, ${ }^{78}$ um dadurch Lust zu bekunden und dem Liebhaber zu schmeicheln. ${ }^{79}$ Indem die matrona aber ausgerechnet Vogelnamen wählt, ${ }^{80}$ spielt sie - ohne sich dessen bewusst zu sein - auf das Verhältnis zwischen Lucius und der Sklavin Photis an, welches Lucius' ungewollte Verwandlung in einen Esel statt in einen Vogel am Anfang des Romans auslöste. Die Kosenamen erinnern gleichzeitig auch an das erotische Verhältnis zwischen Frauen und Spatzen bzw. Tauben, die (u.a. als Metaphern für das männliche Glied) Gegenstand von Catulls und Martials Gedichten sind. ${ }^{81}$

Zoophilie erscheint in Apuleius' Geschichte über den Esel Lucius und die matrona ohne moralische Wertung als mögliche Form der Lustbefriedigung und dient mit der detaillierten Beschreibung der nackten Körper und des Liebesakts der Erotisierung der Lesers. ${ }^{82}$ Wie in der Geschichte über Lucius und die Sklavin Photis, die als kompositorisches Gegenstück zur Liebesnacht zwischen Lucius und der matrona gilt,, ${ }^{83}$ ist die Unterordnung des Mannes unter den Willen der Frau, also die Umkehrung der traditionellen Geschlechterrollen in einer Art servitium amoris, für den Protagonisten mit erotischem Gewinn verbunden. ${ }^{84}$ Durch die Betonung der Bedeutung der Sprache in der Beziehung zwischen Frau und Esel wird gleichzeitig in humorvoller Weise eine gewisse Durchlässigkeit der Grenzen zwischen Mensch und Tier suggeriert, die ebenso wie der Verweis auf die mythische Folie der Pasiphaë-Geschichte, vor der die Episode zwischen matrona und Esel gelesen werden soll - das Anstössige an der Schilderung mildert, und gleichzeitig die Rückverwandlung des Protagonisten in einen Menschen am Ende des Romans vorbereitet.

\section{Schluss}

Zoophilie ist - anders als bisweilen in der Forschung behauptet ${ }^{85}$ - ein Phänomen, das sich in der antiken Literatur nicht nur auf göttlicher Ebene im Mythos, sondern auch zwischen Mensch und Tier findet. In den zoologischen Schriften von Plutarch, Plinius dem Älteren und Aelian wird Zoophilie, die vom Tier initiiert wird, deutlich positiver bewertet als Zoophilie, die vom Menschen ausgeht. Dabei wird das typisch aktive bzw. passive Rollenverhalten bei der erotischen Werbung analog zum Verhältnis zwischen Mann und Frau gezeichnet: In der überwiegenden Mehrheit der Fälle sind es männliche Tiere, die aktiv Frauen oder Knaben erobern. Umgekehrt ergreifen im antiken Roman ausschliesslich Frauen die Initiative zum sexuellen Kontakt mit Tieren. Geschichten über Zoophilie haben hier jedoch anders als bei den Zoologen keinen 
moralisierenden, sondern einen unterhaltenden und erotisierenden Zweck; die zoophilen Frauen werden daher für ihre Taten nicht kritisiert oder bestraft.

\section{BIBLIOGRAPHIE}

Alexandridis, Annetta : „Wenn Götter lieben, wenn Götter strafen : Zur Ikonographie der Zoophilie im griechischen Mythos“, in : A. Alexandridis, M. Wild, L. Winkler-Horacek (Hrsg.), Mensch und Tier in der Antike. Grenzziehung und Grenzüberschreitung, Wiesbaden 2008, 285-311. Armstrong, Rebecca : Cretan Women. Pasiphae, Ariadne, and Phaedra in Latin Poetry, Oxford 2006. Beagon, Mary : Roman Nature. The Thought of Pliny the Elder, Oxford 1992. Beagon, Mary : The Elder Pliny on the Human Animal. Natural History, Book 7, Oxford 2005. Beetz, Andrea : Love, Violence, and Sexuality in Relationships between Humans and Animals, Aachen 2002.

Bruneau, Philippe : „Illustrations antiques du Coq et de l'Âne de Lucien“, BCH 89 (1965) 349-357.

Camilloni, Maria Teresa : „Su la leggenda di Pasifae“, RCCM 28 (1986) 53-66.

Clarke, John R. : Looking at Lovemaking. Constructions of Sexuality in Roman Art 100 B.C.-A.D. 250, Berkeley 1998.

Coleman, K. M. : „Fatal Charades : Roman Executions Staged as Mythological Enactments“, JRS 80 (1990) 44-73.

Coleman, Robert : Vergil Eclogues, Cambridge 1977.

Dekkers, Midas : Geliebtes Tier. Die Geschichte einer innigen Beziehung, München 1994.

Dierauer, Urs : Tier und Mensch im Denken der Antike, Amsterdam 1977.

Dierauer, Urs : „Raison ou instinct ? Le développement de la zoopsychologie antique“, in : Barbara Cassin, Jean-Lous Labarrière (éds.), L'animal dans l'antiquité, Paris 1997.

Dierichs, Angelika : Erotik in der Römischen Kunst, Mainz 1997.

Dierichs, Angelika : Erotik in der Kunst Griechenlands, Antike Welt 24 (1993), Sonderheft.

Dumont, Jacques : Les animaux dans l'Antiquité grecque, Paris 2001.

Elder, J.P. : „Non iniussa cano ; Virgil's Sixth Eclogue“, in : Philip Hardie (ed.), Virgil. Volume I : General Articles and the Eclogues, London/New York 1999, 390-403.

Fögen, Thorsten : „Antike Zeugnisse zu Kommunikationsformen von Tieren“, A\&A 53 (2007) 3975.

Franco, Cristiana : „Riflessioni preliminari per uno studio su animali e costruzione di genere nel mondo antico“, in : A. Alexandridis, M. Wild, L. Winkler-Horacek (Hrsg.), Mensch und Tier in der Antike. Grenzziehung und Grenzüberschreitung, Wiesbaden 2008, 265-284.

Frécaut, Jean-Marc : „L'épisode de Pasiphaé dans l'Art d'aimer d'Ovide (I, 289-326)“, Caesarodunum 17 bis (1982) 17-30. 
French, Roger : Ancient Natural History. Histories of Nature, London/New York 1994.

Gilhus, Ingvild : Animals, Gods and Humans. Changing Attitudes to Animals in Greek, Roman and Early Christian Ideas, London/New York 2006.

Goguey, Dominique : Les animaux dans la mentalité romaine, Bruxelles 2003.

Gourevitch, Danielle : „Sur une image de bestialité“, LMS 5 (1983) 115-129.

Hindermann, Judith : Der elegische Esel. Apuleius' Metamorphosen und Ovids Ars Amatoria, Frankfurt a.M. etc. 2009.

Hindermann, Judith : „The elegiac ass : The concept of servitium amoris in Apuleius' Metamorphoses “, Ramus 38.1 (2009 b) 75-84.

Hindermann, Judith : „Verliebte Delphine, schwimmende Inseln und versiegende Quellen beim älteren und jüngeren Plinius : mirabilia und ihre Erzählpotenz (epp. 4,30;8,20;9,33)“, Gymnasium 118 (2011) 1-10.

Hollis, Adrian S. : Ovid. Ars amatoria. Book I. Edited with an Introduction and Commentary, Oxford 1977.

Hübner, Wolfgang : „Der Mensch in Aelians Tiergeschichten“, A\&A 30 (1984) 154-176.

Johns, Catherine : Sex or Symbol : Erotic Images of Greece and Rome, London 1982.

Kilmer, Martin F. : Greek Erotica on Attic Red-Figure Vases, London 1993.

Krebs, Walter : „Zur kultischen Kohabitation mit Tieren im Alten Orient“, Forschungen und Fortschritte 37 (1963) 19-21.

Krenkel, Werner : Naturalia non turpia : Sex and Gender in Ancient Greece and Rome. Schriften zur antiken Kultur- und Sexualwissenschaft, hrsg. von Wolfgang Bernard und Christiane Reitz, Hildesheim etc. 2006.

Kullmann, Wolfgang : „Zoologische Sammelwerke in der Antike“, in : Wolfgang Kullmann, Jochen Althoff, Markus Asper (Hrsg.), Gattungen wissenschaftlicher Literatur in der Antike, Tübingen 1998, 121-139.

Lang, Dominik : Sodomie und Strafrecht : Geschichte der Strafbarkeit des Geschlechtsverkehrs mit Tieren, Frankfurt a.M. etc. 2009.

Liechti, Martin (Hrsg.) : Die Würde des Tieres, Erlangen 2002.

Lissarague, François : „The Sexual Life of Satyrs“, in : David M. Halperin, John J. Winkler, Froma I. Zeitlin (eds.), Before Sexuality. The Construction of Erotic Experience in the Ancient Greek World, Princeton 1990, 53-81.

Lloyd, Geoffrey E.R. : Science, Folklore and Ideology, Cambridge 1983.

Martini, Wolfram : „II. Griechische Antike“ ; „III. Römische Antike“, in : Peter Dinzelbacher (Hrsg.), Mensch und Tier in der Geschichte Europas, Stuttgart 2000, 29-144.

Massen, Josef : Zoophilie. Die sexuelle Liebe zu Tieren, Köln 1994.

Naas, Valérie : Le projet encyclopédique de Pline l'Ancien, Rom 2002.

Newmyer, Stephen T. : Animals, Rights and Reason in Plutarch and Modern Ethics, New York 2006.

Ramsay, G.G. : Juvenal and Persius, Cambridge ${ }^{1} 1918$, revised and reprinted 1990.

Reckford, Kenneth J. : „Phaedra and Pasiphae : The pull backward“, TAPhA 104 (1974) 307-328. 
Richlin, Amy (ed.) : Pornography and Representation in Greece and Rome, New York 1992.

Robson, James E. : „Bestiality and Bestial Rape in Greek Myth“, in : Susan Deacy, Karen F. Pierce (eds.), Rape in Antiquity, London 1997, 65-96.

Skinner, Marilyn B. : Sexuality in Greek and Roman Culture, Oxford 2005.

Stettner, M. : „Unzucht mit Tieren - ein Tierschutzproblem“, Deutsche Tierärztliche Wochenschrift 97 (1990) 171-174.

Wildberger, Jula : Ovids Schule der „elegischen“ Liebe. Erotodidaxe und Psychagogie in der Ars amatoria, Frankfurt a.M etc. 1998.

Zimmerman, Maaike : Apuleius Madaurensis Metamorphoses. Book X. Text, Introduction and Commentary, Groningen 2000.

\section{NOTES}

1. Mein Dank gilt der Herausgeberschaft von Dictynna und den anonymen Lesern, die mir wertvolle Hinweise und Verbesserungsvorschläge gegeben haben.

2. Vgl. dazu Lang (2009); Beetz (2002) 192-199; Stettner (1990) 173-174.

3. Dekkers (1994) 172-175; 198-199; Massen (1994) 11-16; Beetz (2002) 162.

4. Der bislang umfassendste Beitrag zur Zoophilie im antiken Mythos ist in Zusammenhang mit dem Thema Vergewaltigung und der Frage nach den sozialen Faktoren, welche die Mythen geprägt haben, entstanden (Robson 1997). Vgl. auch Bruneau (1965) und Gourevitch (1983) mit Belegstellen. $\mathrm{Zu}$ den (kultischen) Tier-Mensch-Mythen aus verschiedenen Kulturkreisen und Epochen vgl. Hdt. 2,46; Strab. 17,1,19; Pind. fr. 167 sowie Krebs (1969); Dekkers (1994); Massen (1994) 77-109; Lang (2009) 35-45; Beetz (2002) 164-165.

5. Vgl. z.B. Skinner (2005) 83; Krenkel (2006) 133-134.

6. Vgl. z.B. Richlin (1992); Clarke (1998) 261.

7. In Thorsten Fögens 22-seitiger Bibliographie aus dem Jahr 2006 mit dem Titel „Animals in Graeco-Roman Antiquity and Beyond“ findet sich kein gesonderter Beitrag zur Zoophilie (http:// www.telemachos.hu-berlin.de/esterni/Tierbibliographie_Foegen.pdf). In den Monographien zum Thema Tier wird das Thema meist nur gestreift (z.B. Martini 2000, 52; 84; 142; Goguey 2003, 5960 ) oder mit Fokus auf die geistige Liebe bzw. Freundschaft zwischen Mensch und Tier behandelt.

8. Lang (2009) 47-49.

9. Alexandridis (2008); Franco (2008).

10. Alexandridis (2008) untersucht die Ikonographie der Zoophilie im griechischen Mythos. Vgl. auch Bruneau (1965) zu den Darstellungen von Zoophilie auf römischen Tonlampen. In archäologische Studien wird das Thema öfters aufgegriffen: siehe Johns (1982) 107-113; Dierichs (1993) 115-117 und (1997) 82-83; Kilmer (1993) 57 Anm. 66; 208-209; Lissarague (1990) 61f. und $76 f$.

11. Erwähnt bei Suet. Nero 12,2; 29,1; Mart. De Spect. 5; Apul. met. 10,34,3-35,2; Onos 52-53; vgl. dazu Coleman (1990) 63-69. Siehe auch Catull. 15,18-19.

12. Vgl. dazu Beetz (2002) 166-170.

13. Zeus verwandelt sich in einen Adler, Stier, Schwan, Satyr und in eine Schlange; Poseidon in einen Stier, Adler, Delphin und Schafsbock. Weitere Götter, die in Tiergestalt vergewaltigen, sind Kronos (als Pferd) und Apoll (als Schildkröte und Schlange). In einem zweiten, selteneren Typus von Geschichten behält der vergewaltigende Gott seine menschliche Gestalt, während sein Opfer sich im vergeblichen Versuch, dem Angriff zu entgehen, in ein Tier verwandelt. In einem dritten 
Typus schliesslich treten beide Beteiligte in Tiergestalt auf. Vgl. dazu den Überblick über die verschiedenen Tiermythen mit Belegstellen bei Robson (1997) 74-75; 83-89.

14. So z.B. Helena (Zeus als Schwan und Leda), Athene (Zeus und Metis in wechselnder Tiergestalt), der Schafsbock mit dem Goldenen Vlies (Theophane als Vogel und Poseidon als Schafsbock). Auch Mischwesen können resultieren, so z.B. Cheiron (aus Kronos als Pferd und Philyra) oder der Minotaurus (ein Stier und Pasiphaë). Vgl. dazu Robson (1997) 74-75.

15. Athenaios (deipn. 13,606b-d), dereine Liste bekannter Anekdoten über Tieren bietet, die sich in Menschen verliebt haben, ohne aber weitere Details zu nennen, lasse ich in meiner Untersuchung weg; ebenso Philon (De animalibus 66-67 mit Verweis auf Pasiphaë, Glauke und den Schafsbock sowie den Jungen und den Delphin), der, in der jüdischen Tradition verwurzelt, für die an Zoophilie beteiligten Menschen und Tiere die Todesstrafe fordert (Spec. 3,49-50).

16. Robson (1997) 85 erklärt Ganymeds Entführung durch Zeus in Adlersgestalt als späte Analogiebildung zu ähnlichen heterosexuellen Tier-Mensch-Mythen. Zur Problematik der modernen Termini homo- bzw. heterosexuell vgl. Alexandridis (2008) 287, Anm. 18.

17. Zudem gibt es noch die nur bei Ant. Lib. 21 überlieferte Geschichte der Polyphonte, die als Strafe dafür, dass sie ins Gefolge der Artemis wechselt, von Aphrodite mit Wahnsinn und der Liebe zu einem Bären gestraft wird, dem sie zwei Söhne gebiert.

18. Dies spiegelt sich deutlich in der Sekundärliteratur, wo dieselben Textstellen bezüglich ihres erotischen Gehalts sehr unterschiedlich interpretiert werden, vgl. z.B. Massen (1994) 109-110; dagegen Hübner (1984) 164; French (1994) 21; Dumont (2001) 359.

19. Martini (2000) 84; vgl. auch 59; 142-144; Gilhus (2006) 71-74.

20. Alexandridis (2008) 303.

21. Vgl. dazu Dierauer (1997) 11-17; Lloyd (1983) 14-57; Kullmann (1998) 121-132.

22. Einzig in hist. an. $8,48,631$ a verweist Aristoteles auf die Liebe von Delphinen zu Knaben, ohne

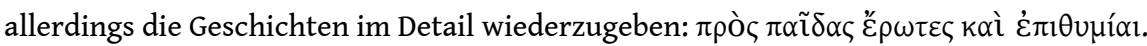

23. So z.B. Hund und Wolf oder Hund und Fuchs, vgl. Aristot. hist. an. 7,28,607a.

24. Vgl.Aristot. GA 4,3,769b; 2,7,746a,29-33. Über den Grund der Entstehung und die verschiedenen Arten von Missbildungen bei Mensch und Tier siehe GA 4,3,769b-771a.

25. Vgl. dazu Kullmann (1983) 129-130; Martini (2000) 74-77; 133-135. Zur Bedeutung der mirabilia bei Plinius dem Älteren siehe Beagon (1992) 8-11; 128-133, 151-152; Naas (2002) 237393.

26. Vgl. zur Konzeption des tierischen Instinkts Dierauer (1997) 20-22.

27. $\mathrm{Zu}$ Plutarchs Auffassung gegenüber der Tierwelt und seiner Auseinandersetzung mit der stoischen Lehre siehe Newmyer (2006) 17-20; 58-65; Dierauer (1977) 253-273; French (1994) 178184; Goguey (2003) 87-89.

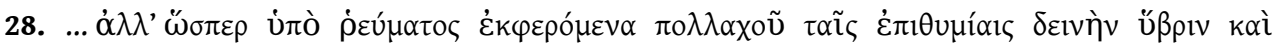

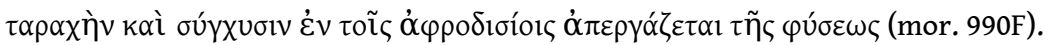

29. Zur moralischen Überlegenheit der Tiere über den Menschen siehe auch mor. 987B; 989A; 990C-D; 493E-F.

30. Vgl. Plin. nat. 10,181-182; Aristot. hist. an. 5,14,546a,21-22; 6,18,572b,23-26.

31. Vgl. Plut. mor. 149C-E über ein Mischwesen, das als Nachkomme zwischen einer Stute und ihrem Wächter beschrieben wird. Als Lehre aus der Geschichte wird gezogen, dass man den Hirten Ehefrauen geben muss. Ebenso berichtet auch Phaedr. 3,3 von Lämmern mit menschlichem Kopf als Resultat der Verbindung von Hirt und Schaf. Vgl. auch Theokr. eid. 1,8688.

32. Plin.nat. 8,155. Zu Plinius' romzentrischer Sichtweise vgl. Beagon (2005) 25-30.

33. Plin. nat. 7,3: eine Frau bringt einen Elefanten zur Welt; eine andere Frau eine Schlange. $\mathrm{Zu}$ Mischwesen vgl. Plin. nat. 9,9-11: Triton, Nereïden, Seemonster; 8,72.75: Sphingen, Pegasi, Mantichora; 5,7: Satyrn, Aegipane. Vgl. zu den Monstren bei Plinius auch Beagon (2005) 46-48; 170-172. 
34. Vgl. z.B. Juv. 2,121-123.

35. Vgl. dazu Liechti (2002); Stettner (1990).

36. Vgl. z.B. Gilhus (2006) 31-35.

37. Vgl. z.B. Colum. 6, praef. 7; Varro rust. 2,5,3-4; Beagon (1992) 151; Martini (2000) 40; 94; 98; 143-144.

38. Vgl. z.B. Sen. epist. 47; Plin. epist. 1,4; 2,6; 5,19; 8,16; 8,19.

39. Phil. De an. 66; vgl. auch Spec. 3,43-45.49. Philon verurteilt Zoophilie ohne zu berücksichtigen, ob sie vom Mensch oder vom Tier ausgeht, und nutzt sie nicht zum Beweis tierischer Intelligenz, sondern reiht sie unter die Laster von Tieren ein.

40. Vgl. Beetz (2002) 195.

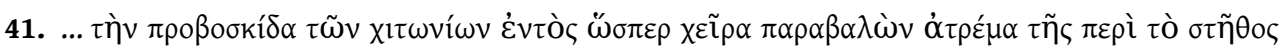

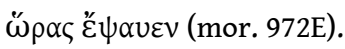

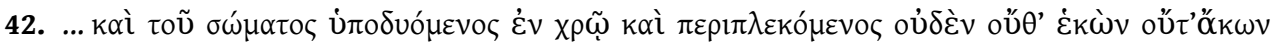
$\varepsilon \Leftarrow \beta \lambda \alpha \psi \varepsilon v$ (mor. 972E).

43. Zwei weitere Geschichten über eine Gans, die einen Knaben liebt, und einen Schafbock, der die Harfespielerin Glauke begehrt (mor. 972F), erwähnt Aristotimus nur dem Titel nach, da sie allgemein bekannt seien.

44. Aristot. part. An. IV, 10 ; 687a7. Vgl. dazu Dierauer (1997) 24-27.

45. Vgl. Dierauer (1997) 6.

46. Dieselbe Geschichte findet sich auch bei Aelian NA 1,38, dort allerdings um zu belegen, dass Elefanten alle Arten von Parfüm, Salben und Blumen lieben. Ähnlich auch in NA 7,43.

47. Vgl. dazu Hindermann (2011).

48. Vgl. Plin. nat. 10,171-172; 7,38.

49. Elefanten z.B. begehen gemäss Plinius (nat. 8,1; 8,12-13) keinen Ehebruch, haben nur im Verborgenen alle zwei Jahre Sex und reinigen sich danach im Fluss, bevor sie zur Herde zurückkehren. Vgl. dazu French (1994) 217-218.

50. Vgl. Fögen (2007) 57-61; Kullmann (1998) 135-136.

51. Hund, Widder, Gans, Dohle, Elefant, Delphin, Schlange, Robbe, Adler, Hahn, Biene.

52. NA 1,$6 ; 12,37 ; 5,29$.

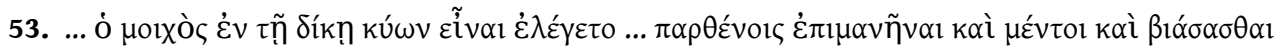
(NA 7,19). Ebenso NA 15,14 über Affen, die umgebracht werden, weil sie mit Frauen Ehebruch begehen.

54. NA 8,$11 ; 6,17$; ähnlich auch in NA 12,39 .

55. Inzesttabu: NA 3,47; 4,7; vgl. dazu auch Aristot. hist. an. 8,47,630b-631a; Nächstenliebe: NA 1,4; 3,23; 5,6; 7,10; Selbstmord aus Scham: NA 3,42.47; 4,7. Tiere werden bei Aelian oftmals als bessere Menschen dargestellt, vgl. dazu Hübner (1984) 161-163.

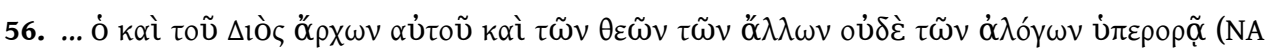
6,17). Ebenso auch NA 1,12.

57. Anthia und Habrokomes bei Xenophon (1,3,1-2); Chariklea und Theagenes bei Heliodor (3,5,4-6); Chaireas und Kallirhoe bei Chariton (1,1,5-7); Leukippe und Kleitophon bei Achilles Tatius (1,4,2-5). Vgl. dazu auch Hübner (1984) 167, der auf die „beinahe monotheistische Omnipräsenz des Eros“ im griechischen Roman verweist.

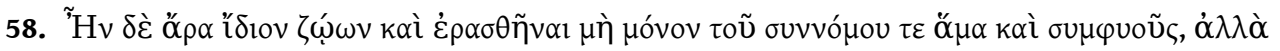

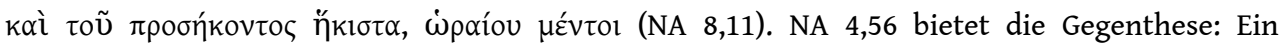
Seehund schläft mit einem äusserst hässlichen Mann und hält ihn für den schönsten aller Menschen. Aelian erklärt dies damit, dass auch Menschen oft hässliche Menschen lieben.

59. Franco (2008) 66-67.

60. In der Loeb-Ausgabe (Ramsay 1990) wird diese Passage in der englischen Übersetzung zensuriert.

61. Vgl. z.B. Mart. 3,81; 4,43; 11,61. 
62. Petron. 137.

63. Im Onos sucht der Protagonist Lukios ganz am Schluss des Romans, wieder in einen Menschen zurückverwandelt, die matrona auf, wird von ihr aber verspottet und weggeschickt, nachdem sie entdeckt, dass sein Penis nun menschliche, und nicht mehr tierische Grösse aufweist.

64. Apul. met. $10,19,4 ; 10,22,5$.

65. nec ullam vaesanae libidini medelam capiens ad instar asinariae Pasiphaae complexus meos ardenter expectabat (Apul. met. 10,19,3)

66. Vgl. dazu Hindermann (2009) 16-20.

67. Vgl. dazu Camilloni (1986); zu den bukolischen Elementen in der 6. Ekloge Elder (1999) 396398.

68. $\mathrm{Zu}$ dieser für eine verheiratete Frau und Mutter ungewöhnlichen Bezeichnung siehe Armstrong (2006) 172; Coleman (1977) 189-190.

69. Vgl. dazu Frécaut (1982); zum Vergleich mit Vergil siehe Hollis (1977) 93-96; Armstrong (2006) 169-186; Wildberger (1998) 90-95.

70. Vgl. dazu Reckford (1974) 320; Hollis (1977) 93.

71. Vgl. dazu auch Prop. 3,19. Zum didaktischen Wert der Erzählungen: Wildberger (1998) 89102.

72. In met. 6,29,4-5 wird Lucius bereits mit dem für Europa in einen Stier verwandelten Jupiter verglichen.

73. Fögen (2007) 39 mit Verweis auf Belegstellen und weiterführende Literatur.

74. Ov. ars 2,703-706.

75. Ov. am. 1,2,35-36; 2,19,17; 3,11a,31-32; ars 2,723-724.

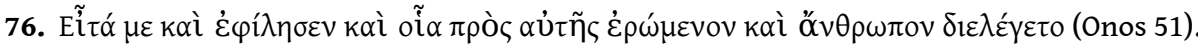

77. Ähnlich auch Ov. ars 3,523-524; am. 3,7,11-12.

78. Ov. ars 3,795-796.

79. Ov. ars 2,689; am. 3,14,25; 1,4,65-66. Ebenso Mart. 10,68; 11,29,3; 11,60,7-8; 11,104,11; Juv. 6,194-197.

80. molles interdum voculas et adsidua savia et dulces gannitus ... iterabat illa et in summa „teneo te“, inquit, „teneo, meum palumbulum, meum passerem“ (met. 10,22,2-3).

81. Catull. 2; 3; Mart. 7,14 (vgl. auch 1,7 und 11,6); siehe dazu Gourevitch (1983) 123-124.

82. Z.B. met. 10,22,1: tam lucida tamque tenera et lacte ac melle confecta membra ... tam vastum genitale; 10,22,4: artissime namque complexa totum me prorsus, sed totum recepit.

83. Vgl. dazu Zimmerman (2000) 26.

84. Vgl. dazu Hindermann (2009b).

85. Siehe z.B.Zimmerman (2000) 265 im Kommentar zum zehnten Buch von Apuleius' Metamorphosen: „Except for this passage and the corresponding passage in the Onos, the only stories in ancient literature that mention sexual acts between humans and animals are mythological ... This makes it all the more remarkable that there are illustrations on lamps ... of a woman coupling with an ass.“ 


\section{RÉSUMÉS}

Die Bedeutung von Zoophilie in der antiken Literatur wurde bislang - mit Ausnahme des Mythos - kaum untersucht. Überblickt man die überlieferten literarischen Texte, fällt auf, dass Zoophilie ausserhalb des Mythos vor allem in zwei literarischen Gattungen auftritt: Einerseits in den zoologischen Schriften von Plutarch, Plinius dem Älteren und Aelian, andererseits im antiken Roman, d.h. in den Metamorphosen des Apuleius und im pseudo-lukianischen Onos. In diesem Beitrag soll untersucht werden, welche Funktion Zoophilie in den beiden literarischen Gattungen hat und wie die Autoren die sexuelle Praktik bewerten, die weder nach griechischem noch römischen Recht strafbar war. Da Vorstellungen über Tiere eng mit Gender und Geschlechterhierarchien verbunden sind, soll insbesondere die Frage berücksichtigt werden, inwiefern männliche und weibliche Verhaltens- und Rollenzuschreibungen bei der Darstellung zoophiler Akte wirksam werden.

INDEX

Mots-clés : Aelian, Apuleius, Liebe, Plinius der Ältere, Plutarch, Roman, Sex, Tier, Zoologie, Zoophilie

\section{AUTEUR}

JUDITH HINDERMANN

Universität Basel

Judith.Hindermann@unibas.ch 